\title{
Macrophages and cathepsin proteases blunt chemotherapeutic response in breast cancer
}

\author{
Tanaya Shree, ${ }^{1,4}$ Oakley C. Olson, ${ }^{1,4}$ Benelita T. Elie, ${ }^{1}$ Jemila C. Kester, ${ }^{1}$ Alfred L. Garfall, ${ }^{1}$ \\ Kenishana Simpson, ${ }^{1}$ Katherine $M$. Bell-McGuinn, ${ }^{1}$ Emily C. Zabor, ${ }^{2}$ Edi Brogi, ${ }^{3}$ \\ and Johanna A. Joyce ${ }^{1,5}$ \\ ${ }^{1}$ Cancer Biology and Genetics Program, ${ }^{2}$ Department of Epidemiology and Biostatistics, ${ }^{3}$ Department of Pathology, \\ Memorial Sloan-Kettering Cancer Center, New York, New York 10065, USA
}

\begin{abstract}
The microenvironment is known to critically modulate tumor progression, yet its role in regulating treatment response is poorly understood. Here we found increased macrophage infiltration and cathepsin protease levels in mammary tumors following paclitaxel (Taxol) chemotherapy. Cathepsin-expressing macrophages protected against Taxol-induced tumor cell death in coculture, an effect fully reversed by cathepsin inhibition and mediated partially by cathepsins $B$ and S. Macrophages were also found to protect against tumor cell death induced by additional chemotherapeutics, specifically etoposide and doxorubicin. Combining Taxol with cathepsin inhibition in vivo significantly enhanced efficacy against primary and metastatic tumors, supporting the therapeutic relevance of this effect. Additionally incorporating continuous low-dose cyclophosphamide dramatically impaired tumor growth and metastasis and improved survival. This study highlights the importance of integrated targeting of the tumor and its microenvironment and implicates macrophages and cathepsins in blunting chemotherapeutic response.
\end{abstract}

[Keywords: cancer; metastasis; chemotherapy; tumor microenvironment; proteolysis; paclitaxel]

Supplemental material is available for this article.

Received January 5, 2011; revised version accepted October 12, 2011.

Solid tumors respond to conventional chemotherapy with many acute changes. In addition to the diverse consequences of massive tumor cell death, these alterations can include collapse of the vasculature and differences in blood flow patterns. Unfortunately, tumors frequently recover from these assaults and re-establish growth. We postulate that there are specific needs for stromal cells and microenvironment-supplied factors under these conditions to enhance tumor cell survival and effect revascularization and remodeling of the extracellular matrix, thus re-establishing a favorable environment for growth. Similar processes at work during different stages of tumor progression have been shown to require the trophic functions of tumor-associated macrophages (TAMs) (Joyce and Pollard 2009; Qian and Pollard 2010). We therefore reasoned that TAMs and their associated products are ideal candidate modulators of response to therapy. TAMs are abundant suppliers of growth factors, cytokines, and proteases, including cysteine cathepsin proteases, which we recently showed to be important

\footnotetext{
${ }^{4}$ These authors contributed equally to this work.

${ }^{5}$ Corresponding author.

E-mail joycej@mskcc.org.

Article is online at http://www.genesdev.org/cgi/doi/10.1101/gad.180331.111.
}

for enhancing pancreatic tumor growth and invasion (Gocheva et al. 2010b). Cysteine cathepsins, or cathepsins as we refer to them here, are a family of 11 members in humans (B, C, H, F, K, L, O, S, L2/V, W, and X/Z), which collectively have important roles in lysosomal protein degradation and make specific individual contributions to pathological conditions, including pancreatitis and cardiomyopathies (Reiser et al. 2010). Here we identified increased levels of specific cathepsin family members in mammary tumors following Taxol treatment and investigated whether this macrophage-supplied activity was functionally relevant in modulating therapeutic response to Taxol and other chemotherapeutic agents.

\section{Results}

\section{Taxol increases cathepsin activity in mammary tumors}

To investigate the response of the tumor microenvironment to chemotherapy, we began by treating MMTVPyMT (PyMT) tumor-bearing mice with maximum tolerated dose (MTD) paclitaxel (Taxol, $50 \mathrm{mg} / \mathrm{kg}$ ), a chemotherapeutic commonly used in breast cancer treatment. PyMT mice develop multifocal lesions in their mammary glands that progress from hyperplastic lesions 
to intraepithelial neoplasias and adenocarcinomas in a typified manner and time course (Lin et al. 2003). In addition, PyMT mice develop lung metastases with high penetrance (Guy et al. 1992). We derived several cell lines from PyMT mammary tumors, including TS1-TGL (Supplemental Fig. S1), which was used for orthotopic implantations and cell-based assays. In both spontaneous and orthotopic models, tumors in Taxol-treated mice grew slower than those in vehicle-treated mice, with differences in tumor volume apparent within 3-7 d (Fig. 1A).

Surveying cathepsin protein levels during Taxol treatment, we found that whole-tumor lysates from Taxolversus vehicle-treated mice had greater levels of cathepsins B, C, and L $7 \mathrm{~d}$ after treatment (Fig. 1B), while other cathepsins did not significantly change (Supplemental Fig. S2A). Quantitative RT-PCR (qRT-PCR) analysis of whole-tumor lysates showed increases in cathepsins B and $S$ at the transcript level and a trend toward increased cathepsin L (Fig. 1C; Supplemental Fig. S2B).

These initial data indicated that Taxol treatment induced an up-regulation of cathepsins in tumors. However, as we examined whole-tumor lysates, this up-regulation could be intrinsic to a specific cell type (for example, tumor cells or macrophages) or due to an increase in proportions of high-cathepsin-expressing cells or both. To discriminate between these possibilities, we first determined whether Taxol directly induced an increase in cathepsin levels in cells by treating PyMT tumor cells in culture with Taxol. We did not find any significant changes in cathepsin expression in Taxol- versus DMSOtreated cells at 24 or $48 \mathrm{~h}$ post-treatment for any of the cathepsin family members expressed (Fig. 1D). To determine whether Taxol induces changes in cathepsin expression in macrophages, we generated primary bone marrow-derived macrophages (BMDMs) from wild-type mice and treated them with Taxol in culture. Again, no detectable increases in cathepsin expression were found for any of the cathepsins expressed by BMDMs at 24 or 48 $\mathrm{h}$ post-treatment (Fig. 1E). We also examined total cathepsin activity levels following Taxol treatment of the BMDMs, given that these cells expressed cathepsins at much higher levels than the tumor cells. BMDM cell lysates were labeled with the biotinylated cathepsin activity-based probe (ABP) DCG04 (Greenbaum et al. 2000), and we found no change in the levels of active cathepsins after Taxol treatment (Supplemental Fig. S2C). These data collectively showed that Taxol treatment results in elevated cathepsin expression and protein in whole tumors, but that this increase is unlikely to be due to a Taxol-mediated cell-intrinsic change in mRNA or activity levels.

\section{Taxol induces an influx of TAMs}

We showed previously that cathepsin activity in untreated PyMT tumors is derived predominantly from TAMs (Gocheva et al. 2010b), and therefore reasoned that cathepsin levels may be higher in Taxol-treated tumors due to increased TAM infiltration. Indeed, image analysis of tissues stained with the macrophage-specific antibody
Iba1 (Kohler 2007; Pyonteck et al. 2011) revealed a notable increase in Taxol-treated tumors, with $53 \%$ more macrophages than their vehicle-treated counterparts $(P<$ 0.0001) (Fig. 2A,B; Supplemental Fig. S3A-C), 7 d after treatment. A similar analysis of vehicle- and Taxoltreated tumors $24 \mathrm{~h}$ after treatment did not reveal any significant differences (Supplemental Fig. S3D), indicating that the response to Taxol occurs after this time point.

These data were corroborated by increased CD68 and CD45 transcript levels in whole-tumor lysates of Taxoltreated tumors as compared with vehicle-treated controls at the 7-d time point (Fig. 2C,D). The increased abundance of TAMs following Taxol treatment did not reflect a general increase in immune cell infiltrates, as costaining for Iba1 and CD45, a pan-leukocyte marker, and quantitation of stained populations revealed that the percentage of $\mathrm{CD} 45^{+} \mathrm{Iba} 1^{-}$cells (i.e., nonmacrophage leukocytes) actually trended toward a decrease in Taxolversus vehicle-treated tumors (Fig. 2E). Thus, increased macrophage infiltration following Taxol is apparently cell-type-specific, rather than due to a general elevation in all infiltrating immune cells. To confirm that macrophages remained the primary source of cathepsin activity after Taxol treatment, we injected Taxol-treated mice with a fluorescent cathepsin ABP as previously described (Gocheva et al. 2010b) and imaged the tumor tissue. We found, as in vehicle-treated tumors, that the majority of cathepsin activity after Taxol treatment was provided by Iba $1^{+}$TAMs (Fig. 2F).

Finally, we examined matched breast cancer patient samples before and after neoadjuvant chemotherapy to determine whether there were similar increases in macrophage numbers. Indeed, we found that in the majority of cases, there was a striking accumulation of macrophages in tumor tissue following chemotherapy compared with those same tumors before treatment (Fig. 2G; patient information summarized in Supplemental Table S1). Costaining with anti-cathepsin antibodies and the anti-CD68 macrophage antibody using multicolor immunohistochemistry revealed a colocalization between cathepsin B or S and CD68 in these samples, confirming that macrophages in these tissues also abundantly expressed these proteases (Supplemental Fig. S4).

\section{Macrophage-derived cathepsins prevent Taxol-induced tumor cell death}

We next asked whether there are any functional consequences of the increase in macrophage infiltration following Taxol and specifically addressed whether macrophages can modulate Taxol-induced tumor cell death. We established an in vitro coculture assay (Fig. 3A) in which the labeled TS1-TGL PyMT cell line (Supplemental Fig. S1) was cultured with syngeneic BMDMs and the coculture was treated with Taxol $24 \mathrm{~h}$ later. Cell death was assayed by flow cytometry $48 \mathrm{~h}$ following treatment. Tumor cells and macrophages were gated using specific markers, and an Annexin V (AnnV)-DAPI protocol was used to identify $\mathrm{AnnV}^{+} \mathrm{DAPI}^{-}, \mathrm{AnnV}^{+} \mathrm{DAPI}^{+}$, and total dead cells $\left(\mathrm{DAPI}^{+}\right)$(Supplemental Fig. S5A). 
A

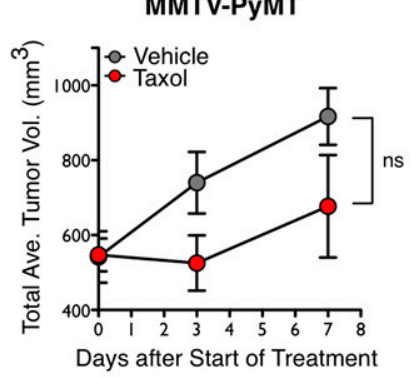

B
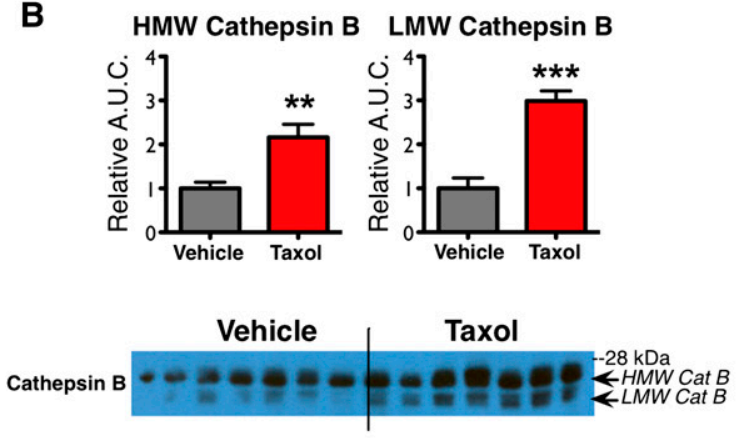

C

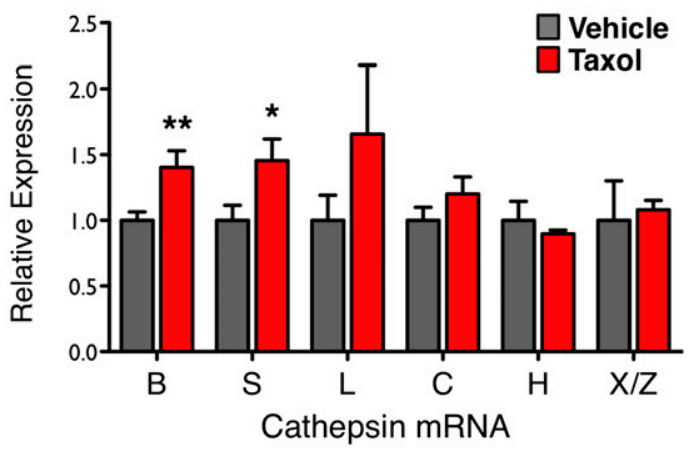

Orthotopically-Implanted

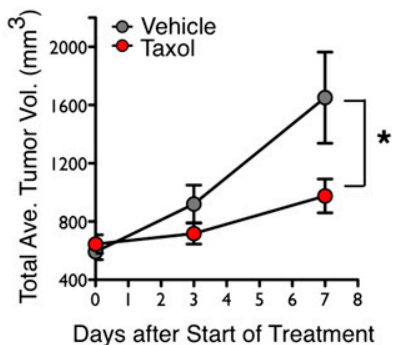

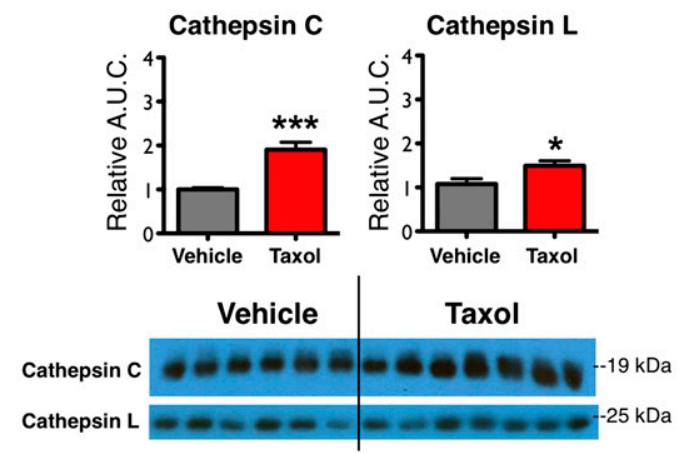

D

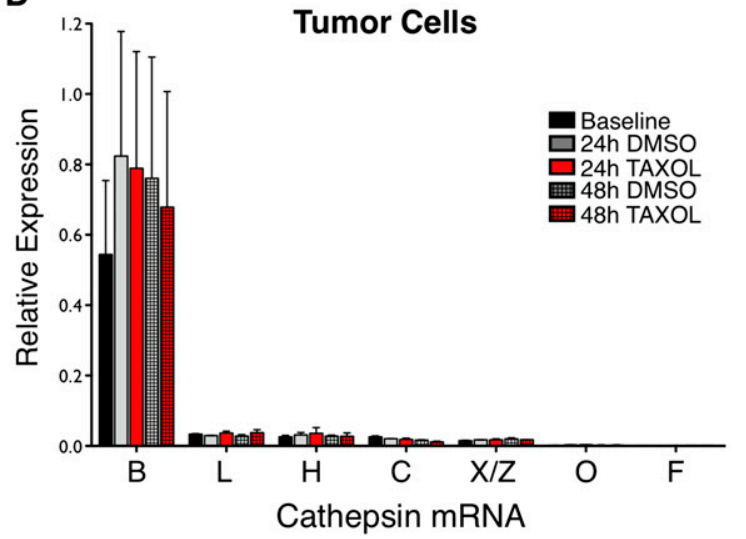

E

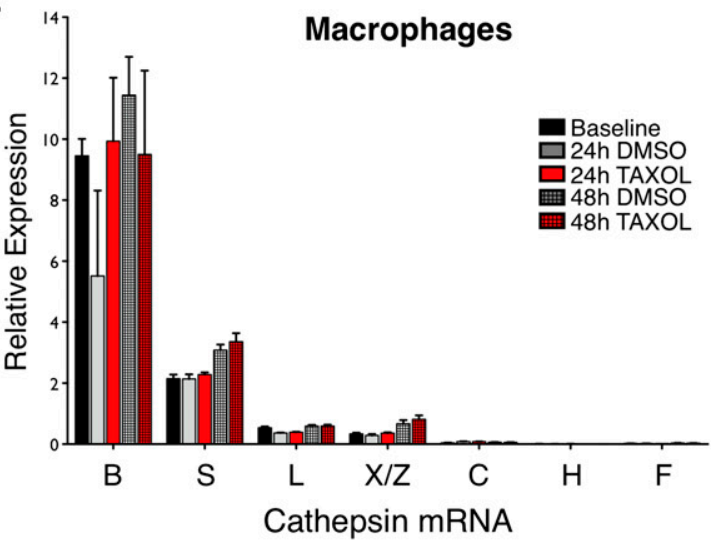

Figure 1. Cathepsin levels in mammary tumors increase following Taxol treatment. $(A)$ Tumor volume curves for PyMT transgenic mice (left) and orthotopically implanted mice (right) treated with one dose of either vehicle or Taxol $(50 \mathrm{mg} / \mathrm{kg}$ ) on day 0, as described in the Materials and Methods. $n=16-17$ for PyMT mice; $n=5-8$ for orthotopic model of TS1 PyMT cell line; $\left({ }^{\star}\right) P<0.05$; (ns) not significant. $(B)$ Taxol increased cathepsin levels at the tumor site. Implanted tumors were harvested from mice $7 \mathrm{~d}$ after vehicle or Taxol treatment, and whole-tumor lysates were assayed for cathepsin proteins. Quantitation of high-molecular-weight (HMW) and lower-molecular-weight (LMW) active cathepsin B (left) and cathepsin C and cathepsin L (right) are shown above the corresponding Western blots. Quantitation of the loading control actin is in Supplemental Figure S2. $n=6-7$ mice per group; $\left.\left(^{\star}\right) P<0.05 ;{ }^{* \star}\right) P<0.01{ }^{\left({ }^{* \star}\right)} P<0.0001 .(C)$ Cathepsin transcripts also increased after Taxol treatment. qRT-PCR analysis for cathepsin genes in lysates from implanted tumors, relative to an endogenous control gene, Ubiquitin C, and normalized to vehicle. $n=7-8$ mice per group; $\left.\left(^{\star}\right) P<0.05,{ }^{\star \star}\right) P<0.01$ by the Mann-Whitney test. $(D, E)$ qRT-PCR analysis of cathepsin transcripts in tumor cells $(D)$ or macrophages $(E)$ in culture following treatment with DMSO control or Taxol showed that expression does not change significantly. $n=3$ independent experiments in each case. 
A

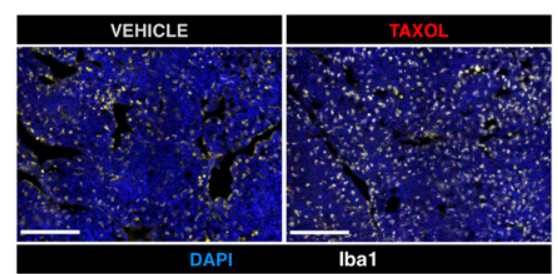

B
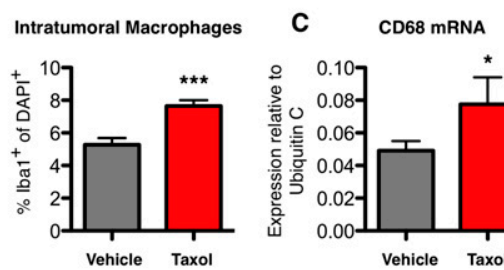

CD45 mRNA

D

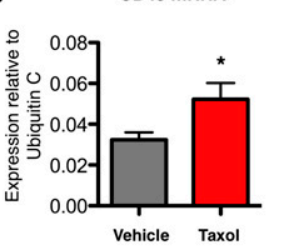

F

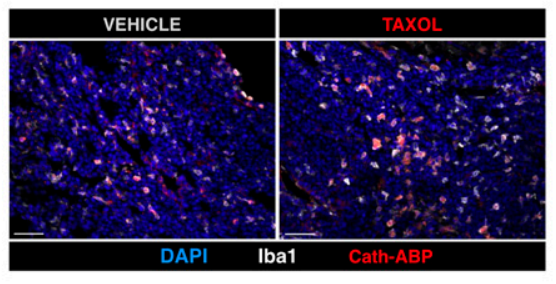

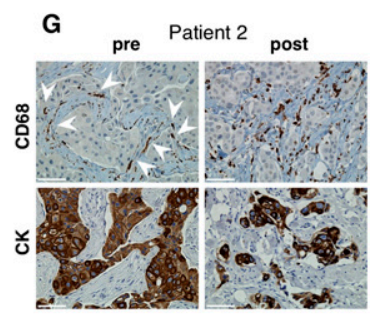

G

pre Patient 3 post

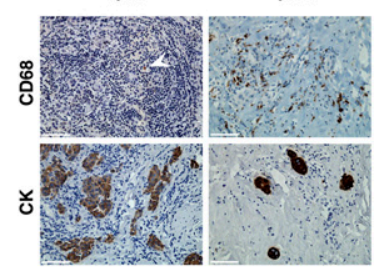

Patient 5 post

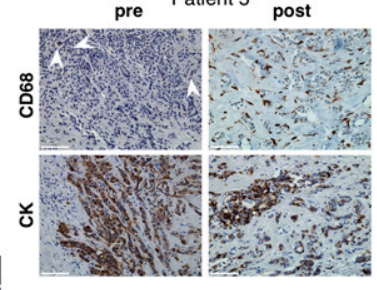

pre Patient 6 post

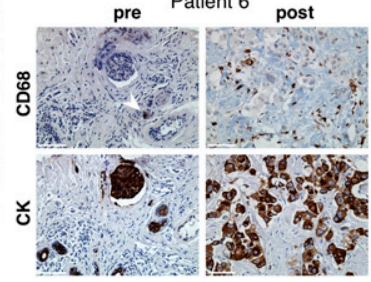

Figure 2. Chemotherapy induces a specific increase in macrophage infiltration in breast tumors. $(A)$ Macrophages accumulate in tumors after Taxol treatment. Representative images of orthotopic tumors stained for the macrophage marker Ibal $7 \mathrm{~d}$ after vehicle or Taxol treatment. Bars, $100 \mu \mathrm{m}$. (B) Quantitation of intratumoral $\mathrm{Iba}^{+}$cells in tumors of vehicle- and Taxoltreated mice via image analysis. $n=12$ vehicle, 9 Taxol; $\left({ }^{\star \star \star}\right) P<0.0001$. All data in $B-F$ are from whole tumors isolated $7 \mathrm{~d}$ after vehicle or Taxol treatment. $(C, D)$ qRTPCR from whole-tumor lysates for transcripts of CD68 (macrophages) and CD45 (all leukocytes) $7 \mathrm{~d}$ after vehicle or Taxol treatment $(n=7-8$ tumors $\left.) ;{ }^{\star}\right) P<$ 0.05. (E) Quantitation by image analysis of the percentage of $\mathrm{CD}_{4} 5^{+} \mathrm{Ibal}^{-}$cells of total $\mathrm{DAPI}^{+}$cells in tumors after treatment with vehicle or Taxol $(n=6$ mice per group). (F) Representative images of tumor sections from mice injected with the cathepsin ABP after vehicle or Taxol treatment and costained with the macrophage marker Ibal. (G) Macrophage numbers increase in breast cancer patients following neoadjuvant chemotherapy. Representative images of matched samples preand post-treatment from patients 2, 3, 5, and 6 stained with CD68 or a pan-cytokeratin (CK) antibody to visualize tumor cells. Positively stained cells are labeled in brown. Rare $\mathrm{CD} 68^{+}$cells in the pretreatment biopsies are indicated by white arrowheads. Patient information can be found in Supplemental Table S1. Bars: $F, G, 50 \mu \mathrm{m}$.

We were intrigued to discover that BMDMs had a potent protective effect on tumor cell death in response to Taxol. While Taxol induced a 3.2-fold increase in death in tumor cells cultured alone $(P=0.0001)$ (Fig. 3B), tumor cells in coculture with BMDMs had substantially lower death rates $(42 \%$ decrease compared with treated monocultures) $(P=0.0041)$ (Fig. 3B). Taxol did not increase death rates in these differentiated macrophages during the course of these experiments (data not shown).

We then sought to determine whether macrophagederived cathepsins participate in the protective effect conferred by these cells in vitro. JPM-OEt (JPM), a pancathepsin inhibitor that inhibits active cysteine cathepsins by binding irreversibly to the catalytic site (Bogyo et al. 2000), was introduced into the coculture system (Fig. 3B). JPM treatment did not influence death of tumor cells or macrophages in monocultures, either alone or in combination with Taxol treatment (Fig. 3; data not shown). However, in the coculture system, cathepsin inhibition completely abrogated the macrophage-conferred protection $(P=0.017)$ (Fig. 3B).

To investigate the broader relevance of these macrophage-protective effects to cell lines other than TS1, we examined a panel of mouse breast cancer cell lines. These included TS2, which was derived from another PyMT primary tumor in our laboratory (Supplemental Fig. S1); Met-1, which was derived from a PyMT lung metastatic lesion (also in the FVB/n background) (Borowsky et al. 2005); and AT-3, a cell line derived from a PyMT primary tumor in the C57BL/6 background (Stewart and Abrams 2007). Macrophages also protected against Taxol-induced death in all cell lines tested $(P<0.05$ to $P<0.01$ for each line compared with Taxol alone) (Fig. 3C), an effect that was significantly abrogated by JPM addition, albeit to different extents $(P<0.05$ to $P<0.01)$ (Fig. 3C).

We next asked whether this protective effect could be attributed to individual cathepsin family members. We modified our coculture assay to include BMDMs derived from Cathepsin B (CtsB)-, CtsC-, CtsL-, or CtsS-null mice, as these family members were up-regulated at the mRNA and/or protein level following Taxol (Fig. 1). We first determined that deficiency for any of these cathepsins did not affect macrophage differentiation in response to colony-stimulating factor-1 (Csf-1) in the derivation protocol (Supplemental Fig. S6A) or impair bead phagocytosis (Supplemental Fig. S6B). Substituting these cells into our assay, we found that while wild-type macrophages consistently protected TS1 tumor cells from Taxol-induced death, macrophages null for CtsB or CtsS were significantly impaired in this ability $(P=0.0448$ and 

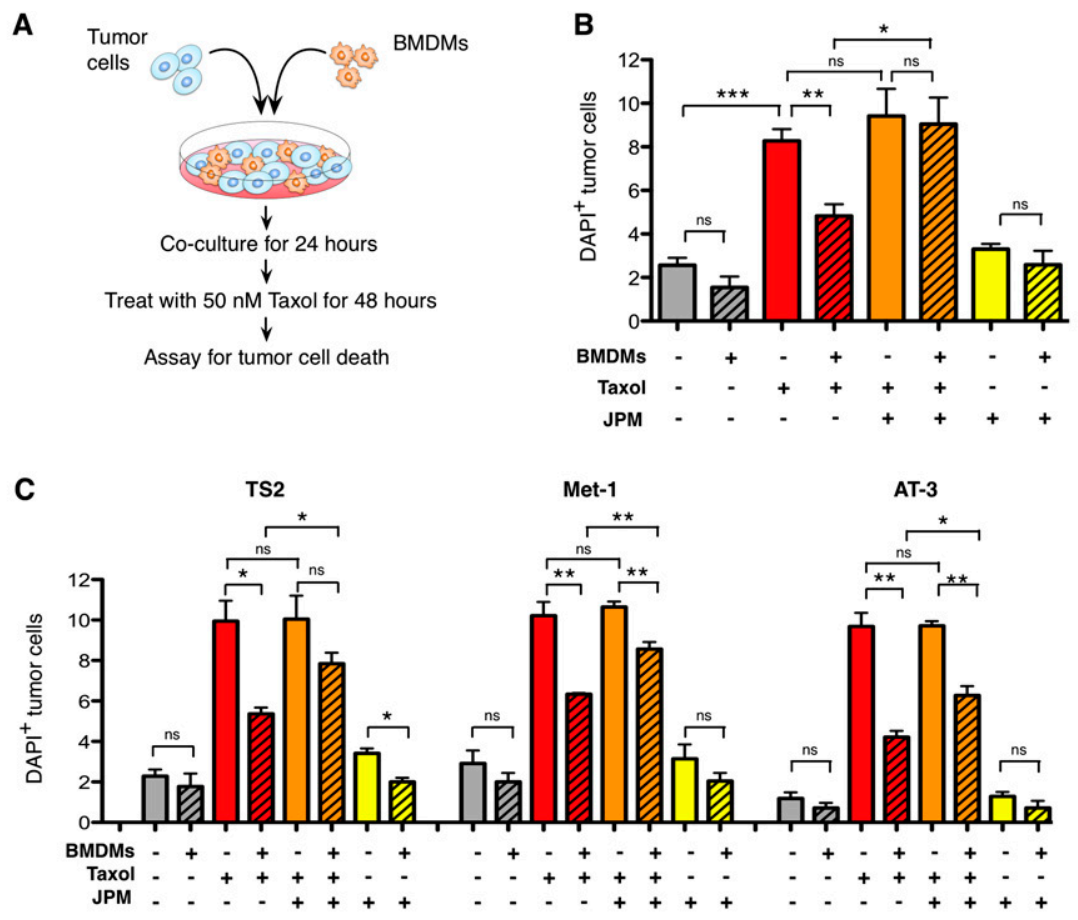

D

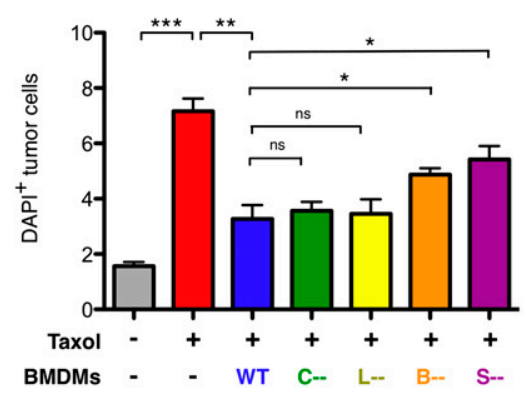

Chemotherapy sensitization by cathepsin inhibition

Figure 3. Macrophage-supplied cathepsins protect tumor cells from cell death. $(A)$ Schematic of coculture assay. (B) Percentage of $\mathrm{DAPI}^{+}$(dead) tumor cells in mono- or coculture of the TS1 cell line with wildtype (WT) BMDMs $48 \mathrm{~h}$ after Taxol (50 nM) or DMSO treatment. Addition of the cathepsin inhibitor JPM $(10 \mu \mathrm{M})$ abrogated the protective effect of BMDMs following Taxol treatment. $(C)$ BMDMs also protected from Taxol-induced cell death in additional PyMT-derived tumor cell lines, TS2, Met1 , and AT-3, with experimental conditions as in $B .(D)$ Percentage of TS1 tumor cell death in monoculture and in cocultures with wild-type or cathepsin B-, cathepsin $S$-, cathepsin $C_{-}$, or cathepsin $L$-null BMDMs $48 \mathrm{~h}$ after treatment. Deletion of cathepsin $B$ or cathepsin $S$ significantly reduced macrophage-conferred protection. (E) Macrophage-conferred protection is largely contact-independent. Cell death in TS1 tumor cells cultured alone or in the presence of wild-type BMDM-CM or CAFCM $48 \mathrm{~h}$ after treatment with Taxol or DMSO control. Addition of JPM $(10 \mu \mathrm{M})$ to the CM abrogated the BMDM-conferred protection. For all graphs, data are from three independent experiments, each done in triplicate. $\left(^{\star}\right) P<0.05 ;\left(^{\star \star}\right) P<0.01 ;\left(^{\star \star \star}\right)$ $P<0.001$; (ns) not significant.
$P=0.0370$, respectively) (Fig. 3D). Macrophages null for Cts C or CtsL, however, still conferred full protection (Fig. 3D), showing that not all macrophage-derived cathepsins participate in this process. The loss of protection observed with individual $C t s B$ or $C t s S$ deletion was not as profound as with JPM treatment, indicating that additional cathepsins are also important for this effect.

Finally, we asked whether the protective effect was dependent on direct contacts between tumor cells and macrophages. To this end, we treated tumor cells and assayed for cell death as before, but substituted BMDMconditioned medium (CM) for direct BMDM coculture (Supplemental Fig. S5B). Interestingly, we found that BMDM-CM recapitulated the protective effect against Taxol-induced tumor cell death $(P<0.0001)$ (Fig. 3E), and the magnitude of protection was similar to that achieved with BMDM coculture. CM from another stromal cell type, a cancer-associated fibroblast (CAF) cell line we derived as reported previously (Abboud-Jarrous et al. 2008), did not affect Taxol-induced cell death, indicating the specificity of this protection by macrophages (Fig. 3E). Addition of JPM to the BMDM-CM abrogated the protective effect $(P=0.0001)$ (Fig. 3E), as we found in the coculture experiments. We verified that JPM completely inhibited cathepsin activity in these experiments (Supplemental Fig. S5C), and used cleaved caspase 3 Westerns as an additional assay of cell death to confirm the flow cytometry results (Supplemental Fig. S5D). From these experiments, we conclude that macrophage-conferred protection against Taxol-induced cell death is largely mediated by secreted factors in a cathepsin-dependent manner.

\section{Cathepsin inhibition sensitizes mammary tumors to chemotherapy}

We next decided to test the hypothesis that the Taxolinduced increases in TAMs, and specifically the cathepsin activity provided by these macrophages, might influence treatment response in vivo. We designed a regression trial in which we treated transgenic PyMT tumor-bearing mice 
with MTD Taxol on days 0 and 17 of a 5 -wk trial and twice daily with the cathepsin inhibitor JPM at $100 \mathrm{mg} / \mathrm{kg}$ per day (Fig. 4A). We initiated treatment when mice reached 9 wk of age, a time at which they typically harbor small but established mammary carcinomas. Four treatment groups were initially examined (Vehicle, Taxol, JPM, and Taxol+JPM).

As expected, Taxol monotherapy produced moderate anti-tumor effects, most evident in the week after each dose was administered $(P<0.01$, area-under-the curve [AUC] comparison with vehicle) (Fig. 4B). JPM treatment did not significantly affect tumor growth (Fig. 4B), despite the abundant cathepsin activity we observed in tumors, the reduction in PyMT tumorigenesis observed when cathepsins $B$ and $Z$ are deleted (Sevenich et al. 2010), and the efficacy of this compound in other tumor models (Joyce et al. 2004). We confirmed that JPM inhibits cathepsin activity in the PyMT tumors using the cathepsin ABP DCG04 $(P=0.0016)$ (Supplemental Fig. S7). This result is somewhat different from a PyMT trial reported by Schurigt et al. (2008), in which there was no significant effect of JPM administration on cathepsin inhibition in the treated tumors. However, the JPM dosing regimen in their study (100 mg/kg per day) involved once-daily administration, compared with twice-daily dosing in the trials herein, which likely accounts for the fact that we observe sustained cathepsin inhibition under these conditions.

Concurrent administration of JPM and Taxol, however, did reveal effects upon combination (Fig. 4B). The addition of JPM slowed tumor growth compared with Taxol treatment alone $(P<0.05$, AUC comparison) (Fig. 4B). In addition to twice-weekly external measurements of tumor volume, tumors were measured upon excision at the end of the trial (Fig. 4E), revealing a significant $58 \%$ decrease in tumor volume in the Taxol+JPM group $(P<0.01$ compared with vehicle). Tumors in Taxol+JPM-treated mice were significantly smaller $(P<0.05)$ than those treated with Taxol alone, indicating a specific advantage of cathepsin inhibition in this context. This combinatorial effect in vivo corroborated the protective effects of macrophagederived cathepsins on Taxol-induced cell death we observed in the culture assays and prompted us to investigate whether we could further improve therapeutic efficacy by including additional treatment modalities.

\section{Low-dose cyclophosphamide (Cyclo) further enhances anti-tumor efficacy}

With the broader goal of simultaneously using multiple anti-tumor therapeutics to further improve efficacy, we added continuous low-dose (CLD) Cyclo to our combination trials. This form of dosing, also termed "metronomic," was devised to achieve better therapeutic efficacy by providing continuous drug exposure with limited toxicity. This dosing regimen has also shown efficacy in clinical trials for patients with metastatic breast cancer (SanchezMunoz et al. 2008). CLD Cyclo has additionally been shown to target the tumor vasculature via direct and indirect effects (Kerbel and Kamen 2004). Cyclo was administered at a low dose $(10 \mathrm{mg} / \mathrm{kg}$ per day) continuously via the drinking water (Fig. 4A), an established route for this drug (Man et al. 2002), and four new groups were added to the trial: Cyclo, Cyclo+JPM, MTD Taxol and CLD Cyclo (Taxol+Cyclo), and Triple (receiving all three drugs).

CLD Cyclo alone had a modest effect on tumor growth $(P<0.05$, AUC compared with vehicle) (Fig. 4C). Addition of cathepsin inhibition to CLD Cyclo did not enhance efficacy (Fig. 4C), in contrast to the effect of adding JPM to MTD Taxol (Fig. 4B). As CLD Cyclo has been reported to have multiple potential anti-tumor mechanisms, including anti-angiogenic effects and inducing tumor cell apoptosis (Pietras and Hanahan 2005; Bell-McGuinn et al. 2007; Blansfield et al. 2008), we investigated both processes in these treated tumors. We found that there was no significant effect on vessel number, length, density, or functionality, as assessed by lectin perfusion, in the CLD Cyclo-treated tumors compared with vehicle at end stage (Supplemental Fig. S8A-D). Analysis of apoptosis by cleaved caspase-3 staining, however, revealed a threefold increase in cell death in the CLD Cyclo group $(P<0.05)$ (Supplemental Fig. S8E). Costaining with either tumor cell (EpCAM) or endothelial cell (CD34) markers showed that the vast majority of apoptotic cells were tumor cells (Supplemental Fig. S8F). This indicates that increased tumor cell death, through either direct or indirect mechanisms, is the process by which CLD Cyclo slows tumor growth in this model.

Taxol+Cyclo treatments combined to produce smaller tumors than vehicle-treated controls $(P<0.0001$, AUC comparison) (Fig. 4D). When we added JPM to treat mice with all three drugs together (Triple), we found that cathepsin inhibition enhanced the chemotherapy combination $(P<0.05$ compared with Taxol+Cyclo; AUC comparison) (Fig. 4D). Measurements of tumors excised at the end of the trial showed significant tumor volume reductions compared with vehicle controls in the following groups: Cyclo $(36 \%, P<0.05)$, Taxol+Cyclo $(63 \%, P<$ $0.0001)$, and Triple $(86 \%, P<0.0001)$ (Fig. 4E). In addition, tumor volumes in the Triple treatment group were significantly reduced compared with the Taxol+Cyclo group (61\% reduction, $P<0.01$ ) (Fig. $4 \mathrm{E}$ ), reflecting a clear benefit of JPM addition. Overall, we found that the most effective treatments were the ones incorporating MTD Taxol and that Taxol monotherapy was substantially improved on by the addition of cathepsin inhibition and CLD Cyclo. At the end of the trial, the Triple combination group had $80 \%$ smaller tumors when compared directly with the Taxol monotherapy group $(P<0.0001)$, a dramatic improvement. Statistical results for all 5-wk trials are summarized in Supplemental Table S2.

One striking observation was that in addition to reducing mean tumor volumes, the combination treatment groups also showed reduced variance in tumor volumes, compared with the vehicle and monotherapy groups. Thus, with the incremental addition of drugs with different targets and mechanisms of action, we achieved increasingly consistent anti-tumor responses rather than additively variable responses. Such effects are difficult to achieve with conventional chemotherapy (Yang et al. 2010) and may represent a reduced propensity for the 
A

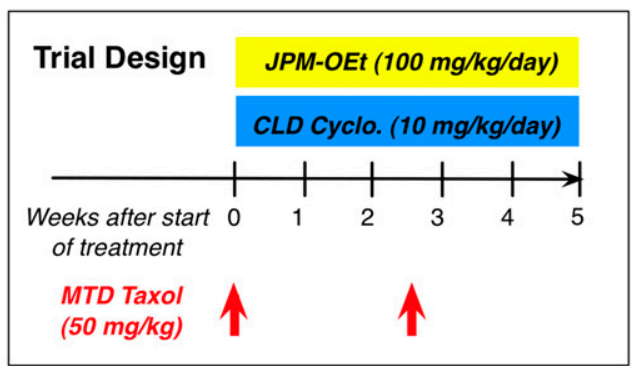

B

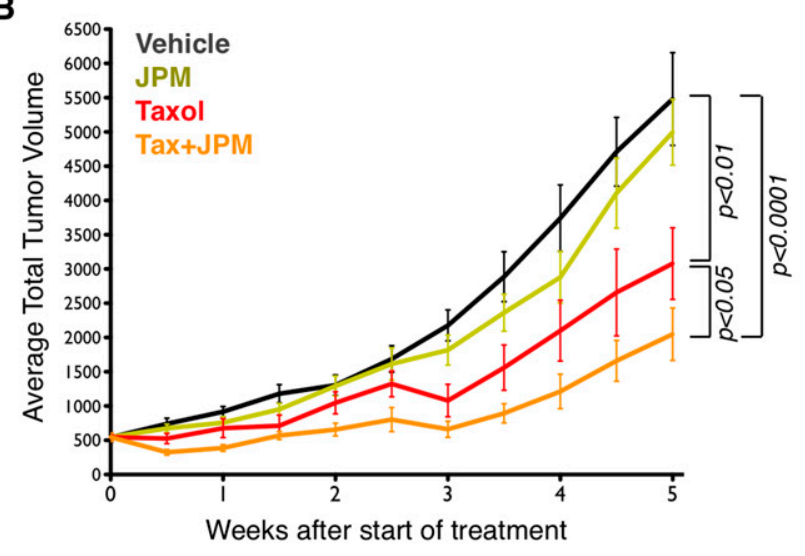

D

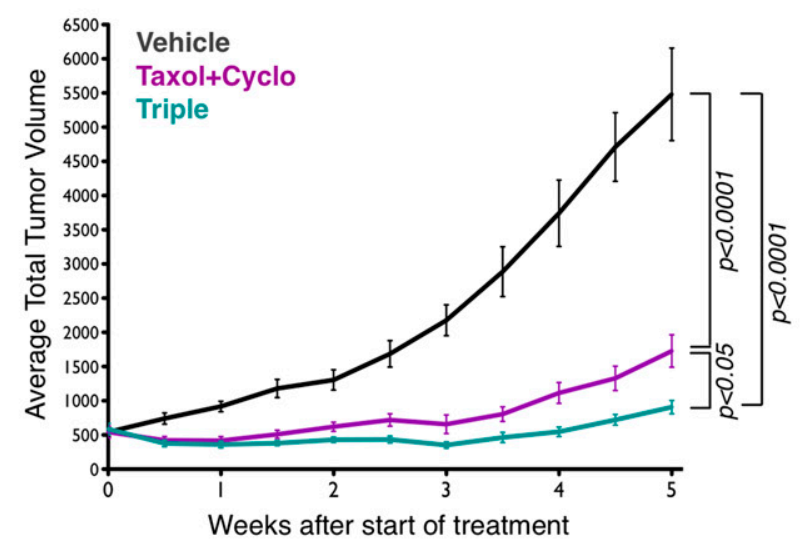

C

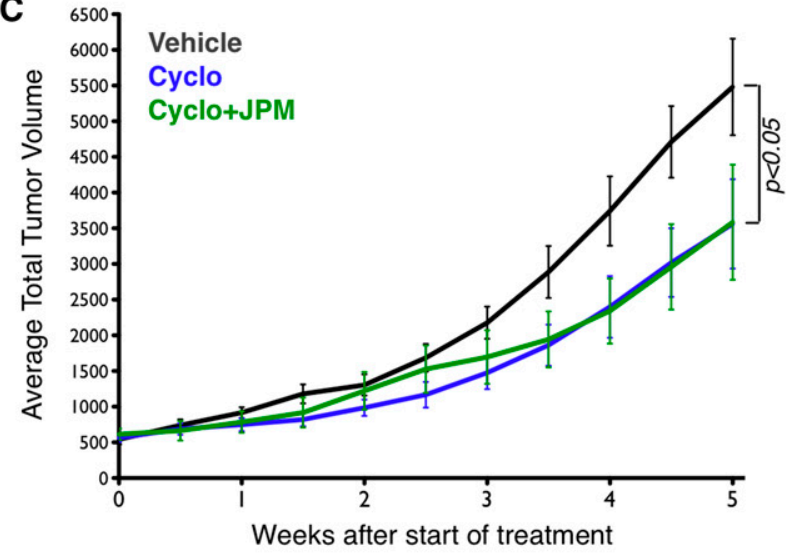

E

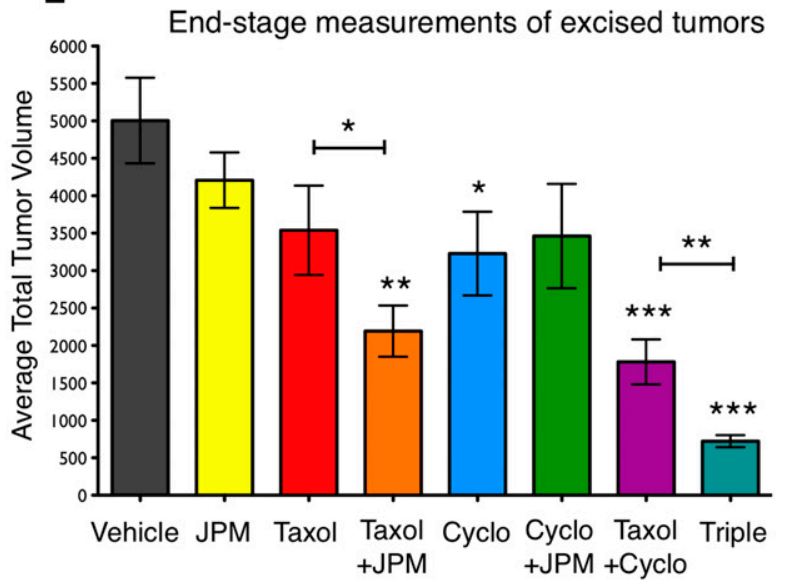

Figure 4. Cathepsin inhibition sensitizes tumors to Taxol treatment. (A) Trial schematic showing dosing of MTD Taxol, JPM, and CLD Cyclo in the 5-wk regression trial (from 9-14 wk of age). (B) Tumor volume curves for PyMT transgenic mice treated with Taxol, JPM, and Taxol+JPM, compared with vehicle, based on twice-weekly external palpation caliper measurements. $n=17$ vehicle, 16 JPM, 16 Taxol, 16 Taxol+JPM. $P$-values in $B-D$ derive from area-under-the-curve (AUC) analyses, with pairwise comparisons between indicated groups, although some of these effects did not reach statistical significance when adjusted for multiple comparisons. (C) Tumor volume curves for Cyclo- and Cyclo+JPM-treated mice compared with vehicle-treated mice. $n=14 \mathrm{Cyclo}, 14 \mathrm{Cyclo}+J \mathrm{PM}$. $(D)$ Tumor volume curves for Taxol+Cyclo- and Triple-treated mice compared with vehicle-treated mice. $n=16$ Taxol+Cyclo, 16 Triple. $(E)$ End-stage tumor volumes based on measurements of excised tumors. $\left(^{\star}\right) P<0.05,\left(^{\star \star}\right) P<0.01,\left(^{\star \star \star}\right) P<0.0001$, unpaired $t$-tests. Comparisons are with the vehicle group unless otherwise indicated. $n=14-15$ per treatment group.

development of acquired chemoresistance when the microenvironment is concurrently targeted.

\section{Combination therapy reduces lung metastatic burden}

We next sought to determine whether secondary metastatic growth in the lung was also impaired by the different treatments. We analyzed serial histological sections from lungs harvested at the end of the trial and measured the total lung metastatic burden in each mouse (Fig. 5A). We found that neither JPM nor Taxol monotherapy significantly reduced median metastatic burden, whereas the combination treatments had a substantial 
A

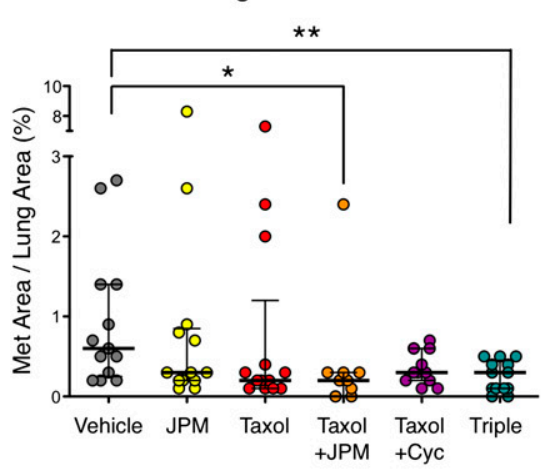

D

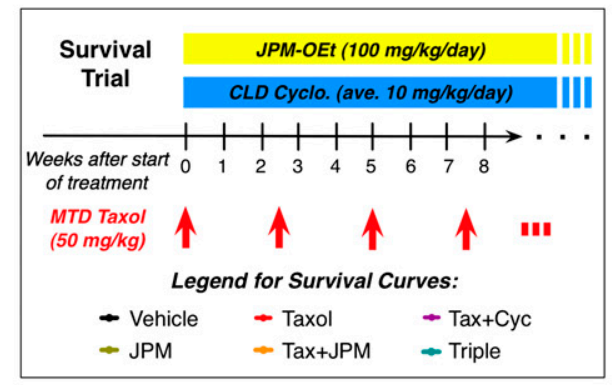

B

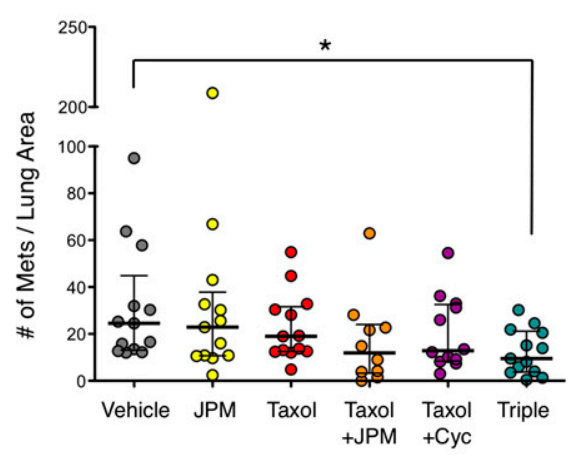

E

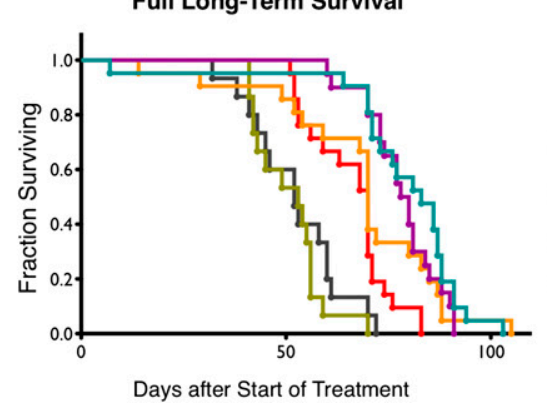

C

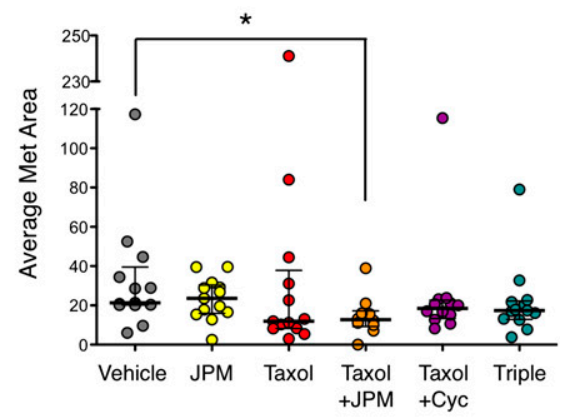

Late Stage Survival

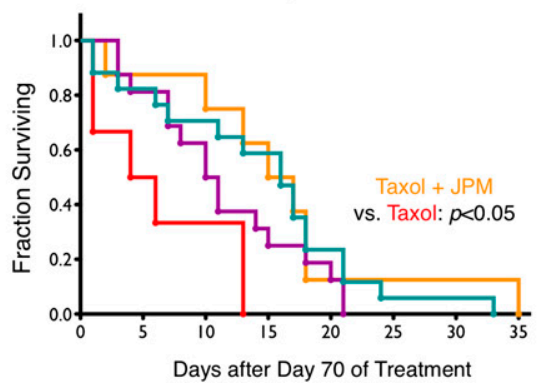

Figure 5. Combination treatments reduce lung metastasis and increase survival. $(A)$ Total lung metastasis burden for indicated treatment groups, as determined by the percentage of total lung area covered by metastases for each mouse. Each data point in the scatter plots represents the mean value for an individual animal; horizontal lines represent the median with the interquartile range. $\left({ }^{\star}\right) P<0.05,\left({ }^{\star \star}\right) P<$ 0.01, Mann-Whitney test; $n=13$ vehicle, 13 JPM, 13 Taxol, 10 Taxol+JPM, 12 Taxol+Cyclo, 13 Triple. (B) Graph showing the number of metastases per mouse per unit area. $(C)$ Graph showing the average size (area in square microns) of metastatic lesions. (D) Survival trial schematic showing dosing of MTD Taxol, JPM, and CLD Cyclo. (E, left) Kaplan-Meier long-term survival curves of mice in the treatment groups indicated. Late-stage survival data beginning at the 70th day of treatment with only mice alive on that day are shown at the right. $n=15-21$ per treatment group. $P$-values from log-rank test comparisons of survival data can be found in Supplemental Table S4.

impact, especially Taxol+JPM $(60 \%$ mean reduction, $P<$ $0.05)$ and the Triple treatment $(73 \%, P<0.01)$, compared with vehicle (Fig. 5A). Statistical results for the lung metastasis analyses are summarized in Supplemental Table S3. Collectively, these data demonstrate that the Triple and Taxol+JPM treatment regimens were highly effective at reducing total lung metastatic burden, likely via effects on incidence $(P<0.05)$ (Fig. 5B) and outgrowth of metastatic lesions $(P<0.05)$ (Fig. 5C), respectively.

\section{Addition of cathepsin inhibition improves late-stage survival}

We next asked whether these combination treatments could also improve survival and designed a long-term study modeled on the original trial, continuing to treat mice every 2.5 wk with MTD Taxol and continuously with CLD Cyclo and JPM (Fig. 5D). Analysis of the full long-term survival curves revealed that all four Taxolreceiving groups had prolonged survival compared with vehicle- and JPM-receiving groups (Fig. 5E). Both Taxol and Taxol+JPM groups had a median survival of $70 \mathrm{~d}$ after treatment initiation, compared with $52 \mathrm{~d}$ for vehicle controls $(P=0.001$ and $P=0.02$, respectively). Taxol+Cyclo treatment and Triple treatment increased median survival to 79 and $83 \mathrm{~d}$, respectively $(P<0.0001$ for both). Again, we found that the addition of CLD Cyclo and JPM to Taxol treatment significantly improved long-term survival $(P<0.0001$, Triple vs. Taxol). Unfortunately, no long-term remissions were observed in this study, and even Triple-treated mice eventually succumbed to their neoplastic disease. As this study was designed as a regression trial, treating mice with already established carcinomas and micrometastases if not macrometastases, we speculate that these combination therapies may yield even better outcomes if begun at an earlier stage of disease or if used in the neoadjuvant setting with subsequent surgical resection of primary tumors. This idea is supported by the observation that combination treatments did initially shrink tumors, rather than simply having a cytostatic effect (Fig. 4).

The complexity of the survival curves and the apparent difference in death rates early versus later in the trial led us to examine these separately. We first truncated the data at $70 \mathrm{~d}$ to focus on early effects of treatment, which were similar to the full survival curves /all statistical results from survival analyses are presented in Supplemental Table S4). To consider survival differences later in 
the course of treatment, we truncated the data to include only mice alive on day 70 and analyzed their survival from this point forward. In this analysis, we found that the Taxol+JPM group fared better than mice receiving Taxol alone $(P<0.05)$ (Fig. 5E). Thus, long-term treatment with combination therapies substantially enhances survival.

\section{Macrophage coculture protects tumor cells from death induced by additional chemotherapeutic drugs}

Having identified a chemoprotective role for macrophages in Taxol-treated tumor cells (Fig. 3), we sought to determine how broadly applicable this finding was. The TS1 tumor cell line was treated with a panel of different drugs with distinct mechanisms of action: etoposide (inhibitor of topoisomerase II activity), doxorubicin (DNA intercalator), gemcitabine (nucleoside ana$\log )$, and carboplatin (DNA-alkylating agent). We found that tumor cell death following etoposide or doxorubicin treatment was reduced by macrophage coculture $(P=0.0076$ and $P=0.0005$, respectively) (Fig. 6A). This protective effect was completely reversed by JPM addition in the case of etoposide-treated cells $(P=0.0064)$ (Fig. 6A) and significantly abrogated in doxorubicin-treated cells $(P=0.0049)$ (Fig. 6A). In contrast, there was no protection conferred against gemcitabine- or carboplatininduced death in the TS1 cell line or any effect of JPM in combination with these drugs (Fig. 6A).

To determine whether the chemoprotective effects were similarly mediated by macrophage-secreted factors, we performed the CM experiments for each drug, as for Taxol above (Fig. 3E). BMDM-CM conferred protection against etoposide- and doxorubicin-induced cell death in TS1 cells $(P=0.0002$ and $P<0.0001$, respectively) (Fig. $6 \mathrm{~B})$, which was significantly abrogated by JPM addition ( $P=0.0010$ and $P=0.0023$, respectively). As in the co-
A

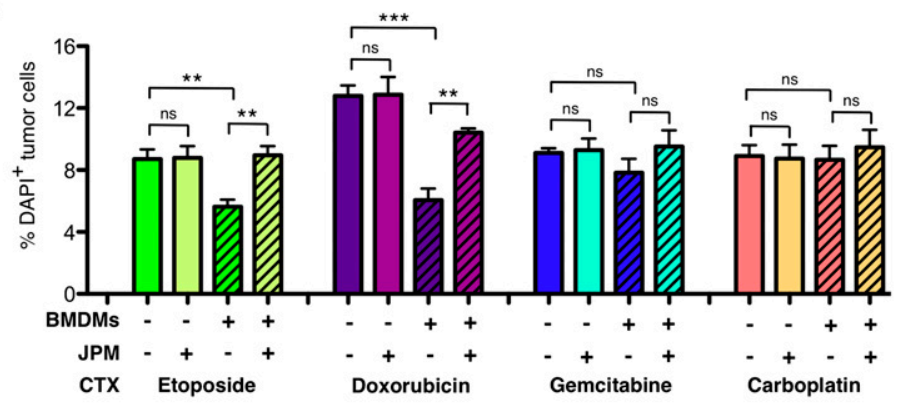

B

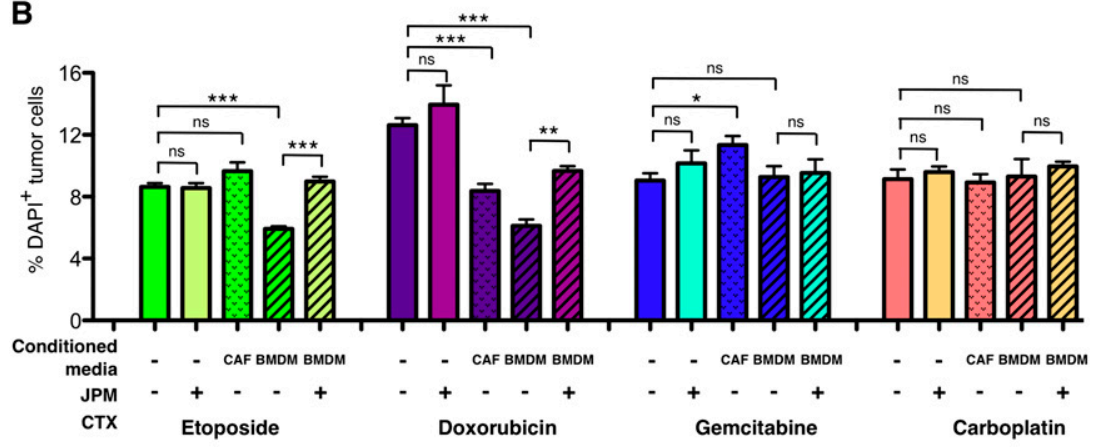

C

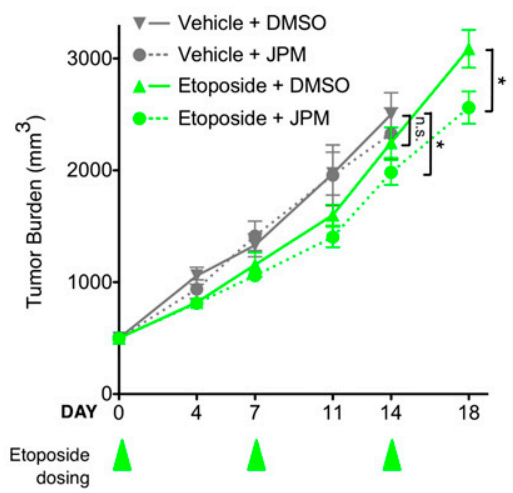

D

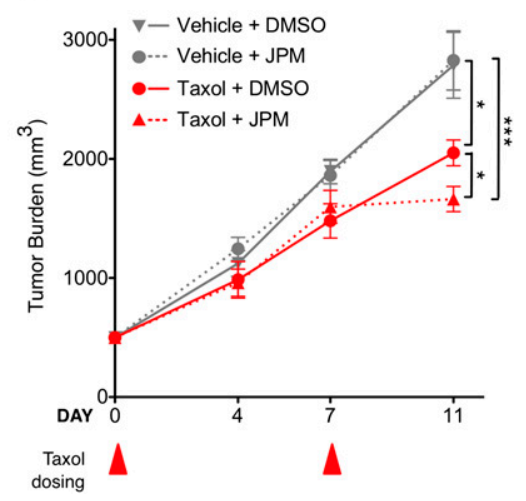

Figure 6. Cathepsin-mediated chemoprotection is relevant to other chemotherapies and additional in vivo models. $(A)$ Percentage of $\mathrm{DAPI}^{+}$(dead) tumor cells in mono- or coculture of the TS1 cell line with BMDMs $48 \mathrm{~h}$ after etoposide $(20 \mu \mathrm{M})$, doxorubicin (300 nM), gemcitabine (400 nM), carboplatin $(50 \mu \mathrm{M})$, or DMSO treatment. Coculture with BMDMs was protective against treatment with etoposide and doxorubicin, but not gemcitabine or carboplatin. As with Taxol, the protective effect observed in BMDM coculture was significantly abrogated by the cathepsin inhibitor JPM (10 $\mu M)$. (B) Cell death in tumor cells cultured alone or in the presence of wild-type (WT) BMDM-CM or CAF-CM $48 \mathrm{~h}$ after treatment with etoposide, doxorubicin, gemcitabine, carboplatin, or DMSO control. For all graphs in $A$ and $B$, data are from three independent experiments, each performed in triplicate. $\left(^{\star}\right)$ $P<0.05 ;\left(^{\star \star}\right) P<0.01 ;\left(^{\star \star \star}\right) P<0.001$; (ns) not significant. (C) $\mathrm{FVB} / \mathrm{n}$ mice orthotopically implanted with TS1 cells were treated with etoposide (10 mg/kg per week). Addition of JPM (100 mg/kg per day) significantly improved response to etoposide. $\left(^{\star}\right) P<0.05$. The etoposide/JPM combination treatment also significantly reduced tumor growth compared with the vehicle-treated group on day 14 (endpoint for the vehicle- and JPMtreated mice due to tumor burden limits). $n=$ 8-10 mice per group. (D) Athy/Nu mice orthotopically implanted with MDA-231 cells were treated with MTD Taxol $(25 \mathrm{mg} /$ $\mathrm{kg}$ per week) or vehicle control. Addition of JPM (100 mg/kg per day) significantly improved response to Taxol in this preclinical xenograft model. $\left(^{\star}\right) P<0.05$ compared with Taxol alone; $\left(^{\star \star \star}\right) P<0.001$ compared with vehicle; $n=9-12$ mice per group. Triangles below the graphs in $C$ and $D$ indicate the time points at which etoposide and Taxol, respectively, were administered. 
culture experiments, there was no effect of BMDM-CM on gemcitabine- or carboplatin-induced apoptosis. Interestingly, while the CAF-CM had no effect on etoposide-, gemcitabine-, or carboplatin-induced cell death, it significantly decreased doxorubicin-induced death $(P=0.0006)$ (Fig. 6B).

We next investigated whether additional tumor cell lines responded similarly and examined the effects of doxorubicin and etoposide on tumor cell death in the presence or absence of macrophage coculture with the TS2, AT-3, and Met-1 cells as above. In all cell lines, and for both drugs, we again observed a significant protective effect when macrophages were included in the coculture $(P<0.05$ to $P<0.01)$ (Supplemental Fig. S9A-C). The extent to which the protective effect was cathepsindependent varied depending on the cell line and the drug used. For the TS2 and Met-1 lines, there was a significant reduction in macrophage protection from etoposide following JPM addition $(P=0.0037$ and $P=0.0177$, respectively) (Supplemental Fig. S9A,B). In contrast to the TS1 line, however (Fig. 3), addition of JPM to these three cell lines did not significantly abrogate the protective effect conferred by macrophages following doxorubicin treatment. Collectively, these experiments show that macrophages confer protection against specific chemotherapies (Taxol, etoposide, and doxorubicin) through cathepsin-dependent and/or cathepsin-independent mechanisms, depending on the particular tumor cell line and drug investigated.

Given that macrophage coculture protected all examined tumor cell lines from etoposide-induced death in a cathepsin-dependent manner, we next asked whether cathepsin inhibition would similarly improve etoposide efficacy in vivo, as we had found for Taxol. We treated mice bearing orthotopic TS1-PyMT tumors with etoposide or vehicle and compared the effects of adding JPM treatment to these two groups. JPM treatment alone had no effect on tumor burden in this trial, consistent with our results in the PyMT transgenic model. However, the addition of JPM to etoposide resulted in a significant decrease in the end-stage tumor volume compared with etoposide alone $(P=0.0297)$ (Fig. 6C). The etoposide/JPM combination treatment also significantly reduced tumor growth compared with the vehicle-treated group on day 14 (endpoint for the vehicle- and JPM-treated mice due to tumor burden limits) $(P=0.0292)$ (Fig. 6C).

We were also interested in determining how patientderived cancer cells would respond to cathepsin inhibition in combination with chemotherapy and thus used a xenograft orthotopic implantation model of the MDAMB-231 breast cancer cell line (Cailleau et al. 1974). Of note, JPM has been previously shown to inhibit both human and mouse cathepsins (Bogyo et al. 2000; Joyce et al. 2004). In this trial, Taxol monotherapy reduced tumor growth compared with either the vehicle- or JPMalone groups $(P=0.0247, P=0.0114$, respectively) (Fig. $6 \mathrm{D})$. This effect was significantly enhanced by the addition of JPM $(P=0.0204$ compared with Taxol, and $P=$ 0.0005 compared with vehicle) (Fig. 6D), demonstrating the synergy between these drugs in a model of human breast cancer. Collectively, these experiments showed that cathepsin inhibition significantly enhanced the efficacy of different therapeutics in coculture and in vivo, an effect we attribute at least in part to the chemoprotective effects of macrophage-supplied cathepsins.

\section{Discussion}

Here we identified a novel adaptive response to chemotherapy wherein macrophages are recruited following cytotoxic chemotherapy and aid in tumor recovery. A similar paradigm for $\mathrm{CD} 11 \mathrm{~b}^{+}$myeloid cells was recently reported in the context of radiotherapy, suggesting broader relevance of this phenomenon (Ahn et al. 2010; Kozin et al. 2010). Whereas several studies have investigated roles for macrophages in amplifying cytotoxic effects of chemotherapy (Nardin et al. 2006; Locher et al. 2010), we found paradoxically that cathepsin-high macrophages are potent suppressors of Taxol-induced tumor cell death, a novel function for them in this context. Interestingly, Taxol has previously been shown to induce mobilization of endothelial progenitor cells from the bone marrow, which are recruited to tumors to aid in revascularization (Shaked et al. 2008). A recent study also reported that paclitaxel induced CSF1-dependent macrophage recruitment and, consequently, that the addition of CSF1R inhibition to paclitaxel significantly enhanced therapeutic efficacy (DeNardo et al. 2011). Therefore, in addition to pleiotropic effects on tumor cells, there is accumulating evidence that Taxol and other chemotherapies alter the tumor microenvironment, as we describe here for breast cancer.

Interestingly, the chemoprotective effects we identified herein for macrophages extend to multiple chemotherapies with different mechanisms of tumor cell killing, yet there is some selectivity to this phenomenon, as not all drugs tested were susceptible to chemoprotection. Chemoprotective effects were identified for Taxol, etoposide, and doxorubicin, which kill tumor cells following microtubule stabilization, inhibition of topoisomerase activity, or DNA intercalation, respectively. These results suggest that macrophages provide survival signals to tumor cells in a cathepsin-dependent manner, which abrogates tumor cell death induced by a variety of different stimuli. Inhibition of cathepsin activity in BMDM-CM at the time of treatment is sufficient to abrogate protection, which strongly points to cathepsins as the macrophagesecreted factors mediating the chemoprotective effect. The identification of the downstream survival signaling pathway(s) is an area of active, ongoing investigation.

Macrophages are clearly emerging as central players in many tumor microenvironments, and cysteine cathepsins are emerging as major effectors of macrophage function in tumors. As such, there is significant potential for targeting macrophages and cathepsins in the treatment of cancer. Cysteine cathepsins are especially attractive targets for a number of reasons. First, they have welldefined catalytic mechanisms against which many inhibitors have been developed (Palermo and Joyce 2008). Second, their activity is increased in many cancers, and more specifically, cathepsins are frequently translocated 
from their intracellular and lysosomal locations to the cell surface and/or secreted into the extracellular milieu in tumors (Mohamed and Sloane 2006). This provides a unique therapeutic opportunity where inhibitors with limited intracellular penetration can specifically target cathepsins in the tumor microenvironment. Finally, targeting macrophage-supplied factors, such as cathepsins, rather than ablating the cells themselves, could represent a more attractive therapeutic strategy, as macrophages are also important for phagocytosis of dead cells, and therefore depleting all macrophage subtypes in combination with cytotoxic therapies may not be desirable.

In conclusion, we showed here that therapeutic interventions can profoundly change the tumor microenvironment and that these alterations can in turn influence the ultimate efficacy of treatment. We found that highdose Taxol treatment increases TAMs in PyMT mice and that macrophage numbers in tumors also increase following chemotherapy treatments in breast cancer patients. These macrophages can in turn act to protect tumor cells from cell death induced by a range of different chemotherapeutic drugs, a cathepsin-dependent function we elucidated through coculture experiments. Cathepsin inhibition during Taxol- or etoposide-based treatment improved anti-tumor efficacy in vivo, demonstrating that simultaneous targeting of microenvironmental-adaptive responses can produce better outcomes and yield more consistent results than conventional chemotherapy, further strengthening the rationale for moving forward with these treatment paradigms in the clinic.

\section{Materials and methods}

\section{Animals and cell lines}

All mice were housed according to institutional standards of the Research Animal Resource Center at Memorial Sloan-Kettering Cancer Center (MSKCC). PyMT mice were obtained from K. Podsypanina and H. Varmus and have been previously described (Guy et al. 1992; Lin et al. 2003). Cathepsin $B^{-/-}$(Halangk et al. 2000) and Cathepsin $L^{-/-}$(Roth et al. 2000) mice in the FVB/n background were obtained from T. Reinheckel. Cathepsin $C^{-/-}$ (Pham and Ley 1999) and Cathepsin $S^{-/-}$(Shi et al. 1999) mice were obtained in the BL/6 background from C. Pham and $\mathrm{H}$. Chapman, respectively, and were backcrossed for $>10$ generations into a pure FVB/n background by V. Gocheva in the Joyce laboratory. Wild-type FVB/n, Athy/Nu, or C57BL/6 animals were purchased from Charles River Laboratories and also bred within our animal facility.

Mice were anesthetized with Avertin and perfused with saline, and tissues were post-fixed in $10 \%$ formalin for an hour before sucrose incubation overnight and OCT (Tissue-Tek) embedding for frozen sectioning. For paraffin embedding, formalin-fixed tissues were processed through an ethanol series into paraffin and embedded. The TS1-TGL cell line was derived from a PyMT mammary tumor, labeled with a triple-imaging TGL vector (Ponomarev et al. 2004), and sorted twice for GFP expression and EpCAM (BioLegend) positivity on a BD FACS Aria. The TS2 cell line was similarly generated in our laboratory from a PyMT primary tumor. Additional tumor cell lines used included Met-1, which was derived from a PyMT lung metastatic lesion (also in the FVB/n background) (Borowsky et al. 2005); AT3 , a cell line derived from a PyMT primary tumor in the C57BL/6 background (Stewart and Abrams 2007); and the MDA-MB-231 cell line (Cailleau et al. 1974). The CAF cell line used for CM experiments was isolated as previously described (AbboudJarrous et al. 2008). For orthotopic implantations, both syngeneic and xenograft, $5 \times 10^{5}$ cells were implanted 1:1 in Matrigel (BD, growth factor-reduced) into the fourth mammary fat pads of wild-type FVB/n or Athy/Nu mice aged 6-9 wk.

\section{BMDMs}

Femurs and tibiae from either $\mathrm{FVB} / \mathrm{n}$ or $\mathrm{C} 57 \mathrm{BL} / 6$ mice were harvested under sterile conditions from both legs and flushed using a 25-gauge needle. The marrow was passed through a 40 $\mu \mathrm{m}$ strainer and cultured in 30-mL Teflon bags (PermaLife) with $10 \mathrm{ng} / \mathrm{mL}$ recombinant mouse Csf-1 (R\&D Systems). Bone marrow cells were cultured in Teflon bags for $7 \mathrm{~d}$, with fresh Csf-1-containing medium replacing old medium every other day to induce macrophage differentiation.

\section{In vitro drug treatments}

For Taxol treatment, cells were treated with $50 \mathrm{nM}$ Taxol (dissolved in medium) or an equivalent amount of DMSO for controls $\left(5 \times 10^{-4} \%\right)$. For cathepsin inhibition, $10 \mu \mathrm{M}$ JPM-OEt was used, with an equivalent amount of DMSO used for controls $(0.01 \%)$. Cells were treated with doxorubicin, etoposide, carboplatin and gemcitabine at concentrations of $300 \mathrm{nM}, 20 \mu \mathrm{M}, 50$ $\mu \mathrm{M}$, and $400 \mathrm{nM}$, respectively. The highest concentration of DMSO $(0.08 \%)$ was used as a vehicle control for the panel of chemotherapies.

\section{Coculture experiments}

Tumor cells and differentiated macrophages were mixed at a 1:1 cell ratio, 200,000 per each cell type, in medium (DME $+10 \%$ FBS) and plated onto 12 -well plates $24 \mathrm{~h}$ prior to treatment. Met1 and AT-3 cells had a significantly increased proliferative rate compared with TS1 or TS2 cell lines and were plated at a density of 100,000 per well. Tumor cells and macrophages were matched for genetic background; FVB/n BMDMs were used for the TS1, TS2, and Met-1 cell lines, while C57BL/6 BMDMs were used for the AT- 3 cell coculture. At the time of treatment, medium was replaced with fresh medium containing Taxol (or other cytotoxic agent) or equivalent DMSO. For experiments involving cathepsin inhibition, JPM was added at the same time as the other agents to the appropriate samples. Forty-eight hours following treatment, cocultures were trypsinized, and all media were collected throughout the experiment to prevent loss of dead cells. Samples were then washed once with FACS buffer (1\% BSA in PBS), followed by Fc block for $15 \mathrm{~min}$ at $4^{\circ} \mathrm{C}$ and antibody labeling for $15 \mathrm{~min}$ at $4^{\circ} \mathrm{C}$. Antibodies used for cell type identification were EpCAM-PE/Cy7 (1:500; BioLegend) and F4/ 80-PE (1:50; Serotec). Samples were then washed in $1 \times$ Annexin V-binding buffer (AVBB; 0.1 M HEPES at $\mathrm{pH} 7.4,1.4 \mathrm{M} \mathrm{NaCl}, 25$ $\mathrm{mM} \mathrm{CaCl}_{2}$ ) and labeled for $10 \mathrm{~min}$ at room temperature with 1.5 $\mu \mathrm{L}$ of Annexin V-APC (BD Biosciences) in $50 \mu \mathrm{L}$ of AVBB. Fifty microliters of AVBB with $1 \mu \mathrm{g} / \mathrm{mL}$ DAPI (Invitrogen) was then added to each sample, followed by acquisition on an LSR II flow cytometer (BD).

\section{CM experiments}

Experiments with $\mathrm{CM}$ were conducted as for the coculture experiments, with CM used in place of macrophages throughout the course of the experiment. Medium that had been conditioned for $24 \mathrm{~h}$ by wild-type BMDMs or CAFs was passed through 0.22 - 
$\mu \mathrm{m}$ filters to remove cellular debris and then added 1:1 with fresh medium to tumor cell monocultures. Controls received fresh medium containing $10 \%$ serum. Where JPM was used, it was added to tumor cell cultures following the same timeline as for the coculture experiments.

\section{Bead phagocytosis assay}

BMDMs were labeled with CellTracker Green CMFDA (Invitrogen) and seeded on coverslips $24 \mathrm{~h}$ before treatment with either $50 \mathrm{nM}$ Taxol or DMSO control. FluoSpheres, $1-\mu \mathrm{m}$ red fluorescent (580/605) carboylate modified microspheres (Invitrogen), were coated overnight in PBS containing $1 \%(\mathrm{w} / \mathrm{v})$ BSA. Twentyfour hours after treatment, microspheres were added at $50 \times$ excess and incubated for $1 \mathrm{~h}$ at $37^{\circ} \mathrm{C}$ or $4^{\circ} \mathrm{C}$. Cells were then washed four times with PBS, and the coverslips were mounted on slides for analysis. Images were visualized under an Axio Imager M1 microscope and acquired using Volocity image acquisition software.

\section{Cathepsin ABP imaging}

Mice were injected with $150 \mu \mathrm{L}$ of a $200 \mu \mathrm{M}$ Cy3b-conjugated cathepsin ABP (Greenbaum et al. 2000; Gocheva et al. 2010b) (synthesized by the Organic Synthesis Facility at MSKCC) intravenously via the tail vein. The probe was allowed to circulate for $1 \mathrm{~h}$ before mice were sacrificed, and tissues were processed as previously described (Gocheva et al. 2010b).

\section{In vivo dosing of drugs}

MTD of Taxol via intraperitoneal (i.p.) injection has been previously determined (Sharma and Straubinger 1994). Accordingly, Taxol (Sigma) was administered i.p. at $50 \mathrm{mg} / \mathrm{kg}$ per dose and dissolved in a 1:1:2 cremophor EL:ethanol:PBS solution (Taxol stock maintained in 1:1 cromophor EL:ethanol, then mixed fresh 1:1 with PBS prior to injection) on day 0 and day 17 for the 5 -wk trials, and every $2.5 \mathrm{wk}$ for the long-term trial. Cyclo (Sigma) was reconstituted to a $20 \mathrm{mg} / \mathrm{mL}$ stock solution in water, $660 \mu \mathrm{L}$ of which was added to $200 \mathrm{~mL}$ of water to place in drinking containers in mouse cages, for a final average dose of $10 \mathrm{mg} / \mathrm{kg}$. This dosing is based on an average daily water consumption of $1.5 \mathrm{~mL}$ of water per $10 \mathrm{~g}$ of body weight per day for mice and has been previously optimized (Man et al. 2002). Fresh Cyclocontaining water was provided to the mice twice weekly. The broad-spectrum cathepsin inhibitor JPM-OEt was synthesized by the MSKCC Organic Synthesis Facility, prepared in a $25 \%$ DMSO solution, and administered twice daily i.p. for a final dose of $100 \mathrm{mg} / \mathrm{kg}$ per day. Vehicle controls in the combination drug trial received equivalent volumes of 1:1:2 cremophor EL:ethanol:PBS solution alone on day 0 and day 17 for the 5 -wk trials or every $2.5 \mathrm{wk}$ for the long-term study, as well as $25 \%$ DMSO twice daily in volumes equivalent to JPM dosing. Etoposide was purchased as a $20 \mathrm{mg} / \mathrm{mL}$ injection solution (TOPOSAR, Teva) and diluted $1: 10$ in $0.9 \%$ sterile saline solution before being administered i.p. at a dose of $10 \mathrm{mg} / \mathrm{kg}$. The etoposide injection solution vehicle consisted of $2 \mathrm{mg} / \mathrm{mL}$ citric acid anhydrous, $80 \mathrm{mg} / \mathrm{mL}$ polysorbate $80,650 \mathrm{mg} / \mathrm{mL}$ polyethylene glycol 300 , and $33.2 \%(\mathrm{w} / \mathrm{v})$ dehydrated alcohol (pH 3.0-4.0).

MMTV-PyMT mice were treated as follows: for Figure 1: from 9-10 wk of age, following one dose of Taxol or vehicle; and for Figures 4 and 5: 9-14 wk of age in the regression trial, and from 9 wk onward in the long-term survival studies, as described in the figure legends. Orthopically implanted mice were treated as follows: For Figure 1, FVB/n mice orthopically implanted with
TS1 cells were treated when tumors reached a total volume of $500 \mathrm{~mm}^{3}$ with a single injection of Taxol $(50 \mathrm{mg} / \mathrm{kg})$ or vehicle, and sacrificed $1 \mathrm{wk}$ later. For Figure 6C, mice were treated with $10 \mathrm{mg} / \mathrm{kg}$ etoposide on days 0,7 , and 14. Etoposide vehicle control was diluted, injected, and dosed in the same manner as the drug arm of the trial. JPM and the equivalent control were administered as described above. For Figure 6D, Athy/Nu mice orthotopically implanted with MDA-MB-231 cells were treated when tumors reached a total volume of $500 \mathrm{~mm}^{3}$ at a determined Taxol MTD of $25 \mathrm{mg} / \mathrm{kg}$. Taxol and vehicle controls were administered on days 0 and 7 as described above.

\section{Combination drug trials}

In all trials, external tumor measurements were made using precision calipers, and tumor volumes (TV) were calculated according to the following formula: $\mathrm{TV}=(a)\left(b^{2}\right) \times \pi / 6(a=$ longest dimension, and $b=$ largest dimensional orthogonal to $a$ ).

Mice were randomized to treatment groups based on their starting tumor burden at $9 \mathrm{wk}$ of age to ensure equivalent distribution among the different groups. At trial endpoints, mice were sacrificed, and tumors were measured again after excision and then processed for further analysis. Mice in the long-term study were monitored twice daily (in conjunction with JPM/ vehicle injections). Endpoints for this study were defined as an individual tumor volume $>3 \mathrm{~cm}^{3}$, a total tumor volume deemed unacceptably burdensome by the animal facility veterinarians, the presence of ulcerated tumors, or signs of obvious distress.

To assess systemic toxicities in the different treatment groups, one to two mice from each group in the 5 -wk trial were submitted to the Comparative Pathology Core Facility at MSKCC. Gross evaluations as well as microscopic and biochemical evaluations of blood, liver, intestine, kidney, skin, and long bones were conducted. Other than occasional gastrointestinal edema and frequent evidence of extramedullary hematopoiesis, found in all groups, their reports found no evidence of drug-related toxicities. In addition, both JPM and low-dose Cyclo have previously been found to be safe, with limited side effects (Emmenegger et al. 2004; Joyce et al. 2004).

\section{Lung metastasis analysis}

Paraffin-embedded left lung lobes for each mouse to be analyzed were serially sectioned, and every 10th slide was stained with hematoxylin and eosin for subsequent blinded analyses. On each slide, metastases were counted and areas were measured for all metastases and for the total lung section. Total lung metastasis burden for each mouse was calculated as the percentage of total lung area analyzed for each mouse that was covered by metastases. Metastasis incidence was calculated as the number of metastases counted in each mouse, normalized to total lung area analyzed per mouse. Average metastasis size was calculated by dividing the total metastasis area calculated for each mouse by the number of metastases discovered in that mouse. All lung metastasis measurements were made using Volocity imaging software.

\section{Protein isolation, labeling, and Western blotting}

Samples were lysed in lysis buffer (5 mM Na-acetate at $\mathrm{pH} 5.5,1$ mM EDTA, 2 mM DTT, 0.1\% Triton X-100), and protein was quantified using the BCA assay (Pierce). Protein lysates were loaded (20 $\mu \mathrm{g}$ per lane) onto SDS-PAGE gels and transferred to PVDF membranes for immunoblotting. Membranes were probed with antibodies against Cathepsin B (1:2000), Cathepsin C (1:1000), Cathepsin H (1:1000), Cathepsin L (1:1000), Cathepsin $\mathrm{S}(1: 1000)$, and Cathepsin X/Z (1:1000) (all from R\&D Systems); 
actin (1:5000; Sigma); cleaved caspase 3 (1:500; Cell Signaling); or GAPDH (1:1000; Cell Signaling) and detected using HRP-conjugated anti-goat or anti-rabbit (Jackson Immunoresearch) antibodies using chemiluminescence detection (Amersham). Bands from Western blots were quantified in the dynamic range using the Gel Analysis module in ImageJ software.

\section{$R N A$ isolation and $q R T-P C R$}

RNA was prepared from samples using TRIzol reagent (Invitrogen) and subsequently DNase-treated. One microgram of RNA was used in cDNA synthesis reactions using the SuperScript III First-Strand system (Invitrogen). Real-time qRT-PCR was performed on cDNA samples using the ABI 7900HT Fast Real-Time PCR system. Primers for cathepsin B (Mm00514439_m1), cathepsin C (Mm00515580_m1), cathepsin F (Mm00490782_m1), cathepsin H (Mm00514455_m1), cathepsin K (Mm00484036_m1), cathepsin L (Mm00515597_m1), cathepsin O (Mm00617413_m1), cathepsin S (Mm00457902_m1), cathepsin W (Mm00515599_m1), cathepsin X/Z (Mm00517697_m1), CD68 (Mm00839636_g1), CD45 (Mm00448463_m1), and Ubiquitin C (Mm01201237_m1) were purchased from Applied Biosystems.

\section{Staining and analysis of tissue sections}

For frozen tissues, $10-\mu \mathrm{m}$ sections were fixed in acetone, preincubated with $1 \times$ PNB-blocking buffer (Perkin Elmer Life Sciences), and incubated with the primary antibody of interest overnight at $4{ }^{\circ} \mathrm{C}$. Appropriate Alexa dye-tagged secondary antibodies (Invitrogen) were used at a 1:500 dilution and incubated for $1 \mathrm{~h}$ at room temperature, followed by incubation in $2 \mu \mathrm{g} / \mathrm{mL}$ DAPI solution (Invitrogen) for $5 \mathrm{~min}$. Species-matched immunoglobulins were used as negative controls. Slides were mounted in ProLong Gold Anti-fade Reagent (Invitrogen), and the tissue sections were visualized under a Carl Zeiss Axio Imager Z1 microscope equipped with an Apotome and AxioCam MRm camera, as well as an automated stage and PixelLink and PCO cameras (TissueGnostics).

The primary antibodies used were as follows: rabbit antimouse Ibal (1:1000; WAKO Chemicals), rat anti-mouse CD34 (1:100; Serotec), rat anti-mouse EpCAM (1:500; eBioscience), rabbit anti-mouse cleaved caspase 3 (1:200; Cell Signaling), and rat anti-mouse CD45 (1:200; Serotec). TissueQuest software (TissueGnostics) was used for colocalization analysis and absolute quantitation of immunofluorescent staining.

For the apoptosis analysis, quantitation was performed using TissueQuest software to determine the percentage of cleaved caspase 3-positive cells per tumor. For these analyses as well as the vasculature quantitation, individual fields of view were stitched using TissueFAXS to cover the entire tumor area. For the angiogenesis analysis, mice were injected with FITC-conjugated lectin as previously described (Gocheva et al. 2006), and tissues were immunofluorescently stained for blood vessels with an anti-CD34 antibody. Vessel area and length were measured using Metamorph software (Molecular Devices), and calculations were performed using established protocols (Xian et al. 2006). Vessel functionality was assessed by calculating the CD $34^{+}$vessel area and the proportion of lectin staining overlapping $\mathrm{CD} 34^{+}$ staining and was quantitated using a Metamorph software macro written by the MSKCC Molecular Cytology Core, as previously described (Gocheva et al. 2010a). The acquisition and analysis of images for all these analyses was performed in a blinded manner.

\section{Patient samples}

Paraffin-embedded samples from core biopsies at the time of diagnosis and excision samples after neoadjuvant chemotherapy at the time of surgical resection were obtained from Dr. Edi Brogi (MSKCC Breast Pathology service). All patient samples were obtained in compliance with the Institutional Review Board at MSKCC. Samples were stained with mouse anti-human CD68 antibody (Dako) at a 1:1 dilution, then further incubated with a biotin-conjugated goat anti-mouse secondary antibody (Vector BA-9200) and the Vectastain ABC kit (Vector Laboratories), followed by detection with SIGMAFAST DAB (Sigma). Cytokeratin staining was performed at the MSKCC Breast Pathology service. For the detection of CD68 in combination with antibodies against cathepsin B or cathepsin S (1:1000 and 1:500, respectively; R\&D Systems), a multiple antigen-labeling method was developed. Briefly, cathepsin B or cathepsin S was visualized using peroxidase substrate and NovaRed detection, followed by anti-CD68 incubation, alkaline phosphatase substrate, and NovaBlue detection. To enable clear visualization of the red/ brown (cathepsin B or cathepsin S) and blue (CD68) double staining, no counterstain was used for these experiments. All images were visualized under a Zeiss Axio Imager Z1 microscope and acquired using AxioVision image acquisition software.

\section{Statistical analysis}

Throughout this study, means \pm SEM /standard error of the mean) are reported unless otherwise specified. For all two-way comparisons, with the exception of tumor volume curves, either unpaired $t$-tests or Mann-Whitney rank sum tests were used. For tumor volume curves, AUC analyses were performed for each mouse and averaged per treatment group, incorporating measurements from the entire $5 \mathrm{wk}$. For the long-term survival study, all comparisons were made using the log-rank (Mantel-Cox) test.

\section{Acknowledgments}

We acknowledge all members of the Joyce laboratory for important contributions to this study, particularly Lisa Sevenich for optimizing the double immunohistochemistry protocol for patient samples and helping with some of the xenograft studies; Vasilena Gocheva for backcrossing CtsC- and CtsS-null mice into the FVB/n background; and Drs. Lisa Sevenich, Alberto Schuhmacher, and Hao-Wei Wang for critical reading of the manuscript. We thank Guangli Li and Ouathek Ouerfelli, MSKCC Synthesis Core, for synthesizing JPM and the cathepsin ABPs; Elyn Reidel, Biostatistics Department, for biostatistics consultations; Ron Blasberg for the triple imaging vector; Thomas Reinheckel for providing CtsB- and CtsL-null mice $(\mathrm{FVB} / \mathrm{n})$; and Xiaoping Chen and Muzaffar Akram for excellent technical assistance. This research was supported by grants from The Breast Cancer Research Foundation (to J.A.J.), Emerald Foundation (to J.A.J.), a NIH ARRA supplement, the Slomo and Cindy Silvian Foundation (to J.A.J. and O.C.O.), the Geoffrey Beene Foundation (to J.A.J. and O.C.O.), and the MSTP and GSK graduate programs (to T.S. and O.C.O.).

\section{References}

Abboud-Jarrous G, Atzmon R, Peretz T, Palermo C, Gadea BB, Joyce JA, Vlodavsky I. 2008. Cathepsin L is responsible for processing and activation of proheparanase through multiple cleavages of a linker segment. I Biol Chem 283: 1816718176.

Ahn GO, Tseng D, Liao CH, Dorie MJ, Czechowicz A, Brown JM. 2010. Inhibition of Mac-1 (CD11b/CD18) enhances tumor response to radiation by reducing myeloid cell recruitment. Proc Natl Acad Sci 107: 8363-8368. 
Bell-McGuinn KM, Garfall AL, Bogyo M, Hanahan D, Joyce JA. 2007. Inhibition of cysteine cathepsin protease activity enhances chemotherapy regimens by decreasing tumor growth and invasiveness in a mouse model of multistage cancer. Cancer Res 67: 7378-7385.

Blansfield JA, Caragacianu D, Alexander HR III, Tangrea MA, Morita SY, Lorang D, Schafer P, Muller G, Stirling D, Royal $\mathrm{RE}$, et al. 2008. Combining agents that target the tumor microenvironment improves the efficacy of anticancer therapy. Clin Cancer Res 14: 270-280.

Bogyo M, Verhelst S, Bellingard-Dubouchaud V, Toba S, Greenbaum D. 2000. Selective targeting of lysosomal cysteine proteases with radiolabeled electrophilic substrate analogs. Chem Biol 7: 27-38.

Borowsky AD, Namba R, Young LJ, Hunter KW, Hodgson JG, Tepper CG, McGoldrick ET, Muller WJ, Cardiff RD, Gregg JP. 2005. Syngeneic mouse mammary carcinoma cell lines: Two closely related cell lines with divergent metastatic behavior. Clin Exp Metastasis 22: 47-59.

Cailleau R, Mackay B, Young RK, Reeves WJ Jr. 1974. Tissue culture studies on pleural effusions from breast carcinoma patients. Cancer Res 34: 801-809.

DeNardo DG, Brennan DJ, Rexhepaj E, Ruffell B, Shiao SL, Madden SF, Gallagher WM, Wadhwani N, Keil SD, Junaid SA, et al. 2011. Leukocyte complexity in breast cancer predicts overall survival and functionally regulates response to chemotherapy. Cancer Discov 1: 52-65.

Emmenegger U, Man S, Shaked Y, Francia G, Wong JW, Hicklin DJ, Kerbel RS. 2004. A comparative analysis of low-dose metronomic cyclophosphamide reveals absent or low-grade toxicity on tissues highly sensitive to the toxic effects of maximum tolerated dose regimens. Cancer Res 64: 3994 4000.

Gocheva V, Zeng W, Ke D, Klimstra D, Reinheckel T, Peters C, Hanahan D, Joyce JA. 2006. Distinct roles for cysteine cathepsin genes in multistage tumorigenesis. Genes Dev 20: $543-556$.

Gocheva V, Chen X, Peters C, Reinheckel T, Joyce JA. 2010a. Deletion of cathepsin $\mathrm{H}$ perturbs angiogenic switching, vascularization and growth of tumors in a mouse model of pancreatic islet cell cancer. Biol Chem 391: 937-945.

Gocheva V, Wang HW, Gadea BB, Shree T, Hunter KE, Garfall AL, Berman T, Joyce JA. 2010b. IL-4 induces cathepsin protease activity in tumor-associated macrophages to promote cancer growth and invasion. Genes Dev 24: 241-255.

Greenbaum D, Medzihradszky KF, Burlingame A, Bogyo M. 2000. Epoxide electrophiles as activity-dependent cysteine protease profiling and discovery tools. Chem Biol 7: 569-581.

Guy CT, Cardiff RD, Muller WJ. 1992. Induction of mammary tumors by expression of polyomavirus middle $\mathrm{T}$ oncogene: $\mathrm{A}$ transgenic mouse model for metastatic disease. Mol Cell Biol 12: 954-961.

Halangk W, Lerch MM, Brandt-Nedelev B, Roth W, Ruthenbuerger M, Reinheckel T, Domschke W, Lippert H, Peters C, Deussing J. 2000. Role of cathepsin B in intracellular trypsinogen activation and the onset of acute pancreatitis. I Clin Invest 106: 773-781.

Joyce JA, Pollard JW. 2009. Microenvironmental regulation of metastasis. Nat Rev Cancer 9: 239-252.

Joyce JA, Baruch A, Chehade K, Meyer-Morse N, Giraudo E, Tsai FY, Greenbaum DC, Hager JH, Bogyo M, Hanahan D. 2004. Cathepsin cysteine proteases are effectors of invasive growth and angiogenesis during multistage tumorigenesis. Cancer Cell 5: 443-453.

Kerbel RS, Kamen BA. 2004. The anti-angiogenic basis of metronomic chemotherapy. Nat Rev Cancer 4: 423-436.
Kohler C. 2007. Allograft inflammatory factor-1/Ionized calcium-binding adapter molecule 1 is specifically expressed by most subpopulations of macrophages and spermatids in testis. Cell Tissue Res 330: 291-302.

Kozin SV, Kamoun WS, Huang Y, Dawson MR, Jain RK, Duda DG. 2010. Recruitment of myeloid but not endothelial precursor cells facilitates tumor regrowth after local irradiation. Cancer Res 70: 5679-5685.

Lin EY, Jones JG, Li P, Zhu L, Whitney KD, Muller WJ, Pollard JW. 2003. Progression to malignancy in the polyoma middle T oncoprotein mouse breast cancer model provides a reliable model for human diseases. Am J Pathol 163: 2113-2126.

Locher C, Conforti R, Aymeric L, Ma Y, Yamazaki T, Rusakiewicz S, Tesniere A, Ghiringhelli F, Apetoh L, Morel Y, et al. 2010. Desirable cell death during anticancer chemotherapy. Ann N Y Acad Sci 1209: 99-108.

Man S, Bocci G, Francia G, Green SK, Jothy S, Hanahan D, Bohlen P, Hicklin DI, Bergers G, Kerbel RS. 2002. Antitumor effects in mice of low-dose (metronomic) cyclophosphamide administered continuously through the drinking water. Cancer Res 62: 2731-2735.

Mohamed MM, Sloane BF. 2006. Cysteine cathepsins: Multifunctional enzymes in cancer. Nat Rev Cancer 6: 764-775.

Nardin A, Lefebvre ML, Labroquere K, Faure O, Abastado JP. 2006. Liposomal muramyl tripeptide phosphatidylethanolamine: Targeting and activating macrophages for adjuvant treatment of osteosarcoma. Curr Cancer Drug Targets 6: 123-133.

Palermo C, Joyce JA. 2008. Cysteine cathepsin proteases as pharmacological targets in cancer. Trends Pharmacol Sci 29: 22-28.

Pham CT, Ley TJ. 1999. Dipeptidyl peptidase I is required for the processing and activation of granzymes A and B in vivo. Proc Natl Acad Sci 96: 8627-8632.

Pietras K, Hanahan D. 2005. A multitargeted, metronomic, and maximum-tolerated dose 'chemo-switch' regimen is antiangiogenic, producing objective responses and survival benefit in a mouse model of cancer. J Clin Oncol 23: 939-952.

Ponomarev V, Doubrovin M, Serganova I, Vider J, Shavrin A, Beresten T, Ivanova A, Ageyeva L, Tourkova V, Balatoni J, et al. 2004. A novel triple-modality reporter gene for wholebody fluorescent, bioluminescent, and nuclear noninvasive imaging. Eur I Nucl Med Mol Imaging 31: 740-751.

Pyonteck SM, Gadea BB, Wang HW, Gocheva V, Hunter KE, Tang LH, Joyce JA. 2011. Deficiency of the macrophage growth factor CSF-1 disrupts pancreatic neuroendocrine tumor development. Oncogene doi: 10.1038/onc.2011.337.

Qian BZ, Pollard JW. 2010. Macrophage diversity enhances tumor progression and metastasis. Cell 141: 39-51.

Reiser J, Adair B, Reinheckel T. 2010. Specialized roles for cysteine cathepsins in health and disease. I Clin Invest 120: 3421-3431.

Roth W, Deussing J, Botchkarev VA, Pauly-Evers M, Saftig P, Hafner A, Schmidt P, Schmahl W, Scherer J, Anton-Lamprecht I, et al. 2000. Cathepsin L deficiency as molecular defect of furless: Hyperproliferation of keratinocytes and pertubation of hair follicle cycling. FASEB J 14: 2075-2086.

Sanchez-Munoz A, Perez-Ruiz E, Ribelles N, Marquez A, Alba E. 2008. Maintenance treatment in metastatic breast cancer. Expert Rev Anticancer Ther 8: 1907-1912.

Schurigt U, Sevenich L, Vannier C, Gajda M, Schwinde A, Werner F, Stahl A, von Elverfeldt D, Becker AK, Bogyo M, et al. 2008. Trial of the cysteine cathepsin inhibitor JPM-OEt on early and advanced mammary cancer stages in the MMTV-PyMT-transgenic mouse model. Biol Chem 389: 1067-1074. 
Sevenich L, Schurigt U, Sachse K, Gajda M, Werner F, Muller S, Vasiljeva O, Schwinde A, Klemm N, Deussing J, et al. 2010. Synergistic antitumor effects of combined cathepsin B and cathepsin $\mathrm{Z}$ deficiencies on breast cancer progression and metastasis in mice. Proc Natl Acad Sci 107: 2497-2502.

Shaked Y, Henke E, Roodhart JM, Mancuso P, Langenberg MH, Colleoni M, Daenen LG, Man S, Xu P, Emmenegger U, et al. 2008. Rapid chemotherapy-induced acute endothelial progenitor cell mobilization: Implications for antiangiogenic drugs as chemosensitizing agents. Cancer Cell 14: 263-273.

Sharma A, Straubinger RM. 1994. Novel taxol formulations: Preparation and characterization of taxol-containing liposomes. Pharm Res 11: 889-896.

Shi GP, Villadangos JA, Dranoff G, Small C, Gu L, Haley KJ, Riese R, Ploegh HL, Chapman HA. 1999. Cathepsin S required for normal MHC class II peptide loading and germinal center development. Immunity 10: 197-206.

Stewart TJ, Abrams SI. 2007. Altered immune function during long-term host-tumor interactions can be modulated to retard autochthonous neoplastic growth. I Immunol 179: 2851-2859.

Xian X, Hakansson J, Stahlberg A, Lindblom P, Betsholtz C, Gerhardt H, Semb H. 2006. Pericytes limit tumor cell metastasis. J Clin Invest 116: 642-651.

Yang R, Niepel M, Mitchison TK, Sorger PK. 2010. Dissecting variability in responses to cancer chemotherapy through systems pharmacology. Clin Pharmacol Ther 88: 34-38. 


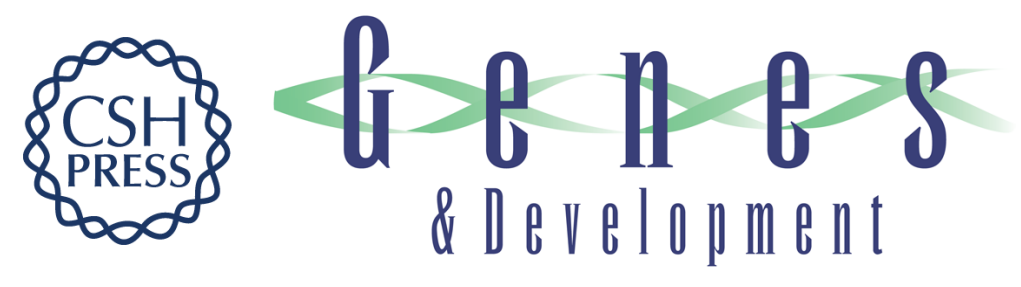

\section{Macrophages and cathepsin proteases blunt chemotherapeutic response in breast cancer}

Tanaya Shree, Oakley C. Olson, Benelita T. Elie, et al.

Genes Dev. 2011, 25:

Access the most recent version at doi:10.1101/gad.180331.111

Supplemental http://genesdev.cshlp.org/content/suppl/2011/12/07/25.23.2465.DC1
Material

References This article cites 41 articles, 15 of which can be accessed free at:

http://genesdev.cshlp.org/content/25/23/2465.full.html\#ref-list-1

License

Email Alerting Receive free email alerts when new articles cite this article - sign up in the box at the top Service 